\title{
CROSS-GLOBAL STUDY ANALYSIS OF THE EFFECT OF COVID-19
}

\author{
Dewi Ratna Sulistina ${ }^{a, *}$, Ernawati Tri Handayani ${ }^{b}$ \\ adewiratnasulistina@gmail.com,dewi.ratna.sulistina-2018@fkm.unair.ac.id \\ ${ }^{a}$ Doctoral Study Program, Faculty of Public Health, Universitas Airlangga, Surabaya, East Java, Indonesia; \\ ${ }^{\mathrm{b}}$ Midwifery D3 Study Program, Universitas Tulungagung
}

\begin{abstract}
Corona Virus Disease-19 (Covid-19) was declared a world pandemic which resulted in thousands of deaths in 216 countries around the world. There are 103 million cases of Covid-19 in the world, with 57.3 million patients are recovered and 2.24 million patients are died. Meanwhile, Indonesia has reported 1,099,687 cases, the highest in Southeast Asia, ahead of the Philippines. In this study, we focused directly on empirical studies and related keywords on the impact of COVID-19 on pregnancy and childbirth. Pregnant women have a higher risk of acquiring respiratory viral infections and severe pneumonia due to physiological changes in the immune and pulmonary systems. The effects of covid-19 on pregnancy and labor were studied and obtained contrary result, obtaining that it has no effect on the occurrence of miscarriages in the early first trimester, pregnant women are not at a higher risk of getting COVID-19 or suffering from more severe diseases than other adults, and no evidence on the ability of virus to be transmitted to the fetus during pregnancy or delivery. In addition, infants and young children are also known to have only mild forms of COVID-19. Meanwhile, other research obtained pro-results; including increased risk of obesity in early pregnancy, increased risk of membranes premature rupture by $11 \%$ and fetal distress by $14 \%$, increased cases of delayed presentation of patients with ectopic pregnancy which causes increased morbidity and mortality. Furthermore, it also causes increased occurrence of hypercoagulability, increased maternal mortality with caesarean section by $58.3 \%$, increased maternal mortality by vaginal delivery by $25 \%$ and premature infant mortality by $16.7 \%$, perinatal mortality and morbidity, high risk of experiencing preeclampsia/eclampsia especially nullipara, severe infection. In addition, studies also discovered that covid19 infection also increased the tendency of admission to intensive care unit, premature birth, risk of severe maternal complications, neonatal complications, miscarriage, stillbirth, fetal growth restriction, FIRS, neurosensory, developmental delay, and mental health improvement in pregnant women. In this case, the alternative management for handling COVID19 during a pandemic in pregnant and maternal women is as follows: vaccines, embryonic mesenchymal stem cells, remdesivir, and convalescent plasma.
\end{abstract}

Keywords : Study Analysis, Effect of Covid-19, Pregnancy and labor 


\section{Introduction}

Corona Virus Disease-19 (Covid-19) was declared a world pandemic which resulted in thousands of deaths in 216 countries around the world. The emergence of this disease at the end of December 2019 in Wuhan (China) was the result of zoonotic transmission (Benarba and Pandiella, 2020). Meanwhile, based on statistical data on Covid-19 cases in the world, it was reported that there were 103 million cases, with the number of patients who recovered was 57.3 million and the number of patients who died was 2.24 million. Meanwhile, Indonesia has reported 1,099,687 cases, the highest in Southeast Asia, ahead of the Philippines. With 30,581 deaths, Indonesia ranks third in Asia and $17^{\text {th }}$ in the world. However, a review of the data suggests that the death toll may be much higher than reported because those who died with acute COVID-19 symptoms but had not been confirmed or tested were not counted in the official death toll. Jakarta Province is the province with the highest number of Covid-19 cases in Indonesia, namely 273,000 cases with 4,312 deaths. Meanwhile, in East Java, 113,000 cases were reported with the highest number of deaths in Indonesia, namely 7,864 cases (Allard, Tom; Lamb, 2021). Coronavirus disease 2019 (COVID-19), caused by the severe acute respiratory syndrome coronavirus 2 (SARS-CoV-2), is a global public health crisis in certain populations, including pregnant women and their newborns. Pregnant women have a higher risk of acquiring respiratory viral infections and severe pneumonia due to physiological changes in the immune system and pulmonary system (Karimi et al., 2021). This study seeks to determine the effect of COVID-19 on pregnancy and labor. The results of this study are expected to contribute to the government in formulating the best approach for appropriate management in handling COVID-19 in pregnancy and labor.

\section{Methods}

This research was conducted based on a survey of general empirical studies on cross-global studies analyzing the impact of COVID-19 on pregnancy and labor. In this study, we conducted a general search under the name "effect of covid-19 on pregnancy and labor". Based on this search we found a large number of article abstracts, which we have read to determine which articles should be included in this research review. After the reading step, most of the articles found is a case study approach and qualitative research analysis. In this study, we focused directly on empirical studies and related keywords on the impact of COVID-19 on pregnancy and labor.

\section{Results and Analysis}

Coronavirus disease 2019 (COVID-19), caused by the severe acute respiratory syndrome coronavirus 2 (SARS-CoV-2), is a global public health crisis in certain populations, including pregnant women and their newborns. Pregnant women have a higher risk of acquiring respiratory viral infections and severe pneumonia due to physiological changes in the immune and pulmonary systems. Based on a general empirical study survey on cross-global studies analyzing the impact of COVID-19 on pregnancy and labor, it is known that COVID-19 causes an $11 \%$ increase in the risk of premature rupture of membranes and $14 \%$ fetal distress during the pandemic. In addition, Covid-19 has no effect on the occurrence of miscarriages in the early first trimester of pregnancy. During the COVID-19 pandemic, it affects the delay in the presentation of patients with ectopic pregnancies, the need for emergency surgical management so that patients are at high risk of experiencing excessive blood loss and increasing morbidity and mortality rates for the mother and fetus. 
Covid-19 also has an effect on increasing D-dimer levels so that pregnant women are at risk for hypercoagulation (blood clots). Furthermore, COVID-19 infection in pregnant women is associated with an increase in mortality of pregnant women undergoing caesarean section by $58.3 \%$, an increase in mortality of pregnant women undergoing vaginal delivery by $25 \%$, and an increase in mortality of premature infants (preterm) by $16.7 \%$. However, pregnant women do not appear to be at a higher risk of developing COVID-19 or developing more severe disease than other adults at the same age. In addition, there is currently no evidence that the virus can be transmitted to the fetus during pregnancy or during delivery. Infants and young children are also known to have only mild forms of COVID-19. Furthermore, Covid-19 was associated with the risk of obesity in early pregnancy occurring in 323 women (48.6\%) with a diagnosis of COVID-19 and 554 women $(40.2 \%)$ without a diagnosis. Women with a diagnosis of COVID-19 were at higher risk for preeclampsia/eclampsia (relative risk [RR], 1.76; 95\% CI, 1.27-2.43), severe infection (RR, 3.38; $95 \% \mathrm{CI}$, 1.63-7.01), admission to the intensive care unit (RR, 5.04; 95\% CI, 3.13-8.10), maternal death (RR, 22.3; 95\% CI, 2.88-172), preterm birth (RR , 1.59; 95\% CI, 1.30-1.94), medical indication for preterm birth (RR, 1.97; 95\% CI, 1.56-2.51), severe neonatal morbidity index (RR, 2 ,66; 95\% CI, 1.69-4.18), and the weight index of perinatal morbidity and mortality (RR, 2.14; 95\% CI, 1.66-2.75). Fever and shortness of breath of any duration were associated with an increased risk of severe maternal complications (RR, 2.56; 95\% CI, 1.923.40) and neonatal complications (RR, 4.97; 95\% CI, 2 ,11-11.69). Asymptomatic women with a diagnosis of COVID-19 remained at higher risk only for maternal morbidity (RR, 1.24; 95\% CI, 1.00-1.54) and preeclampsia (RR, 1.63; 95\% CI, 1, 01-2.63). Among the women who were tested positive (98.1\% by realtime polymerase chain reaction), 54 (13\%) of those neonates were tested positive. Cesarean delivery (RR, 2.15; 95\% CI, 1.18-3.91) but not breastfeeding (RR, 1.10; 95\% CI, 0.66-1.85) was associated with an increased risk of a positive test in neonate. Covid-19 during pregnancy is strongly associated with preeclampsia, especially in nulliparous women. These associations depend on pre-existing risk factors and conditions. The severity of COVID-19 does not appear to be a factor in this association. Both conditions were associated independently of and in additional ways with preterm delivery, severe perinatal morbidity and mortality, and adverse infant delivery. In this case, women with preeclampsia should be considered a group that is particularly vulnerable to the risks posed by COVID-19. Published cumulative data on placentas with COVID-19 infection show common histologic features, which include MVM, inflammation, and FVM. Risks of adverse perinatal and long-term outcomes from COVID-19 infection include miscarriage, stillbirth, fetal growth restriction, early-onset pre-eclampsia, FIRS, neurosensory, and developmental delay. The impact of COVID-19 was associated with more dysfunctional and less emotion-focused coping. COVID-19 stressors, namely financial hardship and social isolation, were associated with more dysfunctional and problem-focused coping. Dysfunctional coping was associated with increased mental health problems and emotion-focused coping was associated with fewer mental health problems. Dysfunctional and emotion-focused coping in part mediate the effects of specific COVID-19 stress on mental health outcomes. The findings suggest that coping is one of the pathways it takes. The COVID-19 pandemic has an impact on mental health in pregnancy. Therefore, support and interventions for pregnant women during the pandemic should focus on improving coping skills to minimize the mental health consequences of COVID-19.

The management of handling covid-19 that can be used as an alternative to handling covid during the pandemic in pregnant and maternal women is as follows: 1) Vaccines Empirical studies related to the safety of vaccines in pregnant women where for the time being this vaccine is not yet mandatory for pregnant women. This vaccine is offered to pregnant women with risk factors of: severe COVID-19 during the launch of the national vaccine program in the UK. This vaccine is also currently offered to pregnant women at high risk of exposure to SARS CoV-2, including health and care workers. In the UK, MHRA receives regular feedback from the vaccination program and any concerns are addressed with appropriate action. So far, no 
safety concerns in vaccinated pregnant women have been reported in either the UK or the US. We believe that COVID-19 vaccination should not be withheld from pregnant women who have received adequate counseling and understand the uncertainty, minimal potential harm, and possible benefits of this vaccine. 2) Embryonic mesenchymal stem cells of advanced mesenchymal cell therapy is one of the immunomodulatory therapeutic approaches currently being explored and may hold promise. It shows that circulating fetal stem cells may have an immune-protective effect on the mother and contribute to the often mild and even asymptomatic postCovid-19 pregnancy. Thus, COVID-19 pregnancy emerges as a paradigm for a more advanced and more comprehensive approach to understand the mechanisms and actions of circulating stem cells in immunoprotection and hypoxia in the microcirculation. 3) Remdesivir and convalescent plasma Remdesivir and convalescent plasma may be effective for the treatment of severe COVID-19 in pregnancy, but additional data are needed to guide risk-benefit considerations. Randomized controlled trials are ongoing and need to be conducted to determine the efficacy of remdesivir and convalescent plasma in the management of severe SARS-CoV-2 infection, but this case and other limited case series show promising results for remdesivir and plasma cures as viable therapies for COVID-19.

\section{Conclusion}

Coronavirus disease 2019 (COVID-19), caused by the severe acute respiratory syndrome coronavirus 2 (SARS-CoV-2), is a global public health crisis in certain populations, including pregnant women and their newborns. Pregnant women have a higher risk of acquiring respiratory viral infections and severe pneumonia due to physiological changes in the immune and pulmonary systems. The effects of covid-19 on pregnancy and labor include: 1) Contrary research: it has no effect on the occurrence of miscarriages in the early first trimester of pregnancy, pregnant women are not at a higher risk of getting COVID-19 or suffering from more severe diseases than other adults, no evidence found concerning that the virus can be transmitted to the fetus during pregnancy or delivery. In addition, infants and young children are also known to have only mild forms of COVID-19. 2) Pro-Research further includes: increased risk of obesity in early pregnancy, increased risk of premature rupture of membranes by $11 \%$, fetal distress by $14 \%$, increased cases of delayed presentation of patients with ectopic pregnancy which has an effect on increasing morbidity and mortality. In addition, covid19 also increased the occurrence of hypercoagulability, maternal mortality with caesarean section by $58.3 \%$, maternal mortality by vaginal delivery by $25 \%$ and premature infant mortality by $16.7 \%$, perinatal mortality and morbidity. Furthermore, pregnant women who are infected with covid-19 are at high risk of experiencing preeclampsia/eclampsia especially nullipara, severe infection, admission to intensive care unit, premature birth, experience increased risk of severe maternal complications, neonatal complications, miscarriage, stillbirth, fetal growth restriction, FIRS, neurosensory, developmental delay, and mental health improvement. Furthermore, the management for handling COVID-19 that can be used as an alternative for handling Covid during a pandemic in pregnant and maternal women is as follows: vaccines, embryonic mesenchymal stem cells, remdesivir, and convalescent plasma.

\section{Acknowledgements}

Faculty of Public Health, Universitas Airlangga, Surabaya, East Java, Indonesia and Midwifery D3 Study Program, Universitas Tulungagung 


\section{References}

Ahmed, E., Dina, E. and Usman, A. (2021) 'The Effect of Remdesivir and Convalescent Plasma in Severe COVID-19 in Pregnancy', Clinical Medical Reviews and Case Reports, 8(5). doi: 10.23937/2378-3656/1410349.

Allard, Tom; Lamb, K. (2021) Exclusive: More than 2,200 Indonesians have died with coronavirus symptoms, data shows. Available at: https://www.reuters.com/article/us-health-coronavirus-indonesia-casualti/exclusive-more-than-2200-indonesians-have-diedwith-coronavirus-symptoms-data-shows-idUSKCN22A04N.

Barg, M. et al. (2021) 'Delayed presentation of ectopic pregnancy during the COVID-19 pandemic: A retrospective study of a collateral effect', International Journal of Gynecology and Obstetrics, 153(3), pp. 457-461. doi: 10.1002/ijgo.13647.

Benarba, B. and Pandiella, A. (2020) 'Medicinal Plants as Sources of Active Molecules Against COVID-19', Frontiers in Pharmacology, 11(August), pp. 1-16. doi: 10.3389/fphar.2020.01189.

Du, M. et al. (2021) 'Association between the COVID-19 pandemic and the risk for adverse pregnancy outcomes: A cohort study', BMJ Open, 11(2). doi: 10.1136/bmjopen-2020-047900.

Kalafat, E. et al. (2021) 'Benefits and potential harms of COVID-19 vaccination during pregnancy: evidence summary for patient counseling', Ultrasound in Obstetrics and Gynecology, 57(5), pp. 681-686. doi: 10.1002/uog.23631.

Karimi, L. et al. (2021) 'Effect of COVID-19 on Mortality of Pregnant and Postpartum Women: A Systematic Review and MetaAnalysis', Journal of Pregnancy, 2021. doi: 10.1155/2021/8870129.

Khoury, J. E. et al. (2021) 'Coping strategies mediate the associations between COVID-19.pdf'

Papageorghiou, A. T. et al. (2021) 'Preeclampsia and COVID-19: results from the INTERCOVID prospective longitudinal study', American Journal of Obstetrics and Gynecology, pp. 1-17. doi: 10.1016/j.ajog.2021.05.014.

Rotshenker-Olshinka, K., Volodarsky-Perel, A. and Steiner, N. (2021) '2_COVID-19 pandemic efect on early pregnancy.pdf'. Archives of Gynecology and Obstetrics, pp. 839-845.

Samara, A. and Herlenius, E. (2021) 'Is There an Effect of Fetal Mesenchymal Stem Cells in the Mother-Fetus Dyad in COVID-19 Pregnancies and Vertical Transmission?', Frontiers in Physiology, 11(February). doi: 10.3389/fphys.2020.624625.

Villar, J. et al. (2021) 'Maternal and Neonatal Morbidity and Mortality among Pregnant Women with and without COVID-19 Infection: The INTERCOVID Multinational Cohort Study', J AMA Pediatrics, pp. 1-10. doi: 10.1001/jamapediatrics.2021.1050.

Wang, C. L. et al. (2021) 'Impact of covid-19 on pregnancy', International Journal of Medical Sciences, 18(3), pp. 763-767. doi: $10.7150 /$ ijms.49923.

Wong, Y. P., Khong, T. Y. and Tan, G. C. (2021) 'The Effects of COVID-19 on Placenta and Pregnancy: What Do We Know So Far?', Diagnostics, 11(1), p. 94. doi: 10.3390/diagnostics11010094.

Zaalishvili, Z. and Pkhaladze, N. (2020) 'EFFECT OF COVID-19 INFECTION ON PREGNANCY-INDUCE HYPERCOAGULABILITY'. 